\title{
Theoretical High Pressure Study for Thermoelastic Properties of NaCl-B1
}

\author{
*Sirwan K. Jalal \\ Adnan M. Al-Sheikh \\ Department of Physics/College of Science/ University of Mosul \\ *Email: sirwan.jalal@ymail.com
}

(Received 2/7/2013;Accepted 28/10/2013)

\begin{abstract}
The evaluation of $\frac{V}{V_{o}}$, Bulk modulus $K$, Lattice parameter $a$, First Grüneisen parameter $\gamma$,
\end{abstract} Second Grüneisen parameter $q$, and Debye temperature $\theta_{D}$ for NaCl-B1 under high pressure has been achieved in this work by using two equations of state (Dodson EOS and Thomsen EOS). The results obtained by the two EOSs have been compared with each other and with other theoretical and experimental data published in literature. The results obtained by using Dodson EOS show more agreement with data given in literature than the results obtained by using Thomsen EOS.

Keywords: High pressure, Thomsen EOS, Dodson EOS, NaCl-B1.

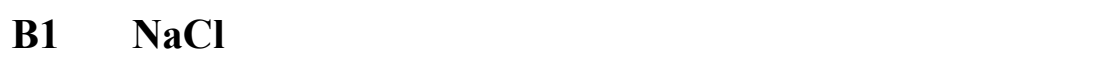

\section{الملخص}

بلستخدلم معادلتي الحالة (Dodson EOS and Thomsen EOS) م لسنقصاء تغير (الحجم النبي ومعلمل المرونة

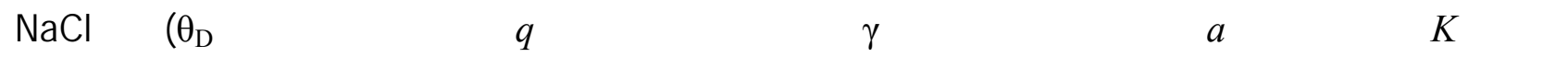

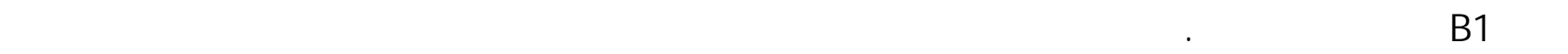

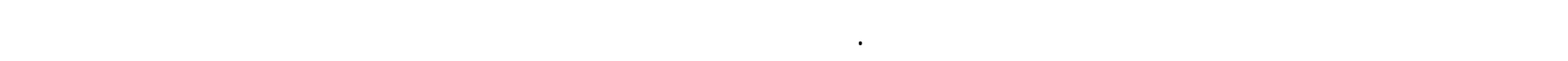
الحالة لـ ـ Dodson كلفت لكثر توافقا، مع البيانلت المنشورة في الأبحلث العلمية، من النتائج التي مم الحصول عليها بلستخدلم

معادلة الحالة J _ Thomsen

الكاملت الدالة: الضغط العالي،معادلة الحالة ل ـ Thomsen، معادلة الحالة J ـ Dodson ، DaCl-B1.

\section{INTRODUCTION}

The determination of pressure - volume - temperature $(\mathrm{P}-\mathrm{V}-\mathrm{T})$ relation of solid materials is a problem of considerable importance in basic and applied science (Recio et al., 1993). The pressure -volume- temperature relationship termed as equation of state (EOS), with the help of EOS, one can determine various properties of crystals under varying conditions of pressures and temperatures (Kumar and Kumar, 2007).

The knowledge of the properties of the simple ionic solids under the conditions of high temperatures and pressures is of fundamental concern to solid-state physics and is important for characterizing the earth's deep interior. The alkali halides are important prototypes for such studies (Chen et al., 2004).

Thermoelastic and thermodynamic properties such as thermal expansivity, bulk modulus, Grüneisen parameter and Debye temperature are of central importance for understanding the behavior of solids (Sun et al., 2008). 
Sodium Chloride is one of the most important materials and a typical ionic solid in highpressure research, used as a pressure gauge in laboratory measurements of compression data (Boehler and Kennedy, 1980; Liu and Bassett, 1973; Decker, 1971) and it has been calibrated extensively through various methods (Brown, 1999; Decker, 1971).

Moreover X-ray diffraction studies can be conveniently made, using devices such as the diamond anvil cell (DAC), thereby affording a useful way of measuring pressure through its effect on the lattice spacing change of simple cubic solids, volume changes at high pressures are measured and, the pressure is estimated using an equation of state. Serious efforts have been made to develop an equation of state of NaCl-B1 phase for its importance as one of the simplest ionic crystals and a commonly used pressure calibrant at pressures below 30GPa (Brown, 1999; Birch, 1986; Decker, 1971).

$\mathrm{NaCl}$ has a stable structure (B1) up to a pressure of about $30 \mathrm{Gpa}$, and its melting temperature is about $1074 \mathrm{~K}$, thus we have a wide range of pressures and temperatures for studying the equation of state and thermoelastic properties of $\mathrm{NaCl}$ (Chauhan and Singh, 2007). $\mathrm{NaCl}$ was used to serve as a solid pressure transmitting medium (Birch, 1986; Klotz et al., 1995).

$\mathrm{NaCl}$ transforms from the $\mathrm{B} 1$ (NaCl-type) to the $\mathrm{B} 2$ (CsCl-type) structure at about 30GPa and $300 \mathrm{~K}$ (Bassett et al., 1968; Sorensen, 1983). The behavior of $\mathrm{NaCl}$ at high pressures is generally described by the isothermal bulk modulus $K_{\mathrm{o}}$ and its pressure derivatives $K_{\mathrm{o}}^{\prime}$ and $K_{\mathrm{o}}^{\prime \prime}$ at atmospheric pressure, the values of these parameters show a considerable disagreement among the many authors partly because they depend on the choice of the equation used to describe the experimental data (Boehler and Kennedy, 1980).

This work proposed evaluating NaCl-Blthermoelastic properties and its phonon frequency spectrum under high pressure by using Dodson and Thomsen EOSs.

\section{Structures of $\mathrm{B} 1$ and $\mathrm{B} 2$ of $\mathrm{NaCl}$}

(Fig. 1) shows the structure of $\mathrm{B} 1$ and $\mathrm{B} 2$ for $\mathrm{NaCl}$. B1-phase is a useful pressure scale only at pressures less than $30 \mathrm{GPa}$, the $B 2$ phase of $\mathrm{NaCl}$ would be more useful as a pressure standard at high pressures since it is stable over wide temperature and pressure ranges up to $3000 \mathrm{~K}$ and far above $100 \mathrm{GPa}$ (Ueda et al., 2008). Values of bulk modulus at atmospheric pressure $K_{\mathrm{o}}$ and its pressure derivatives $K_{\mathrm{o}}^{\prime}$ are listed in Table (1).

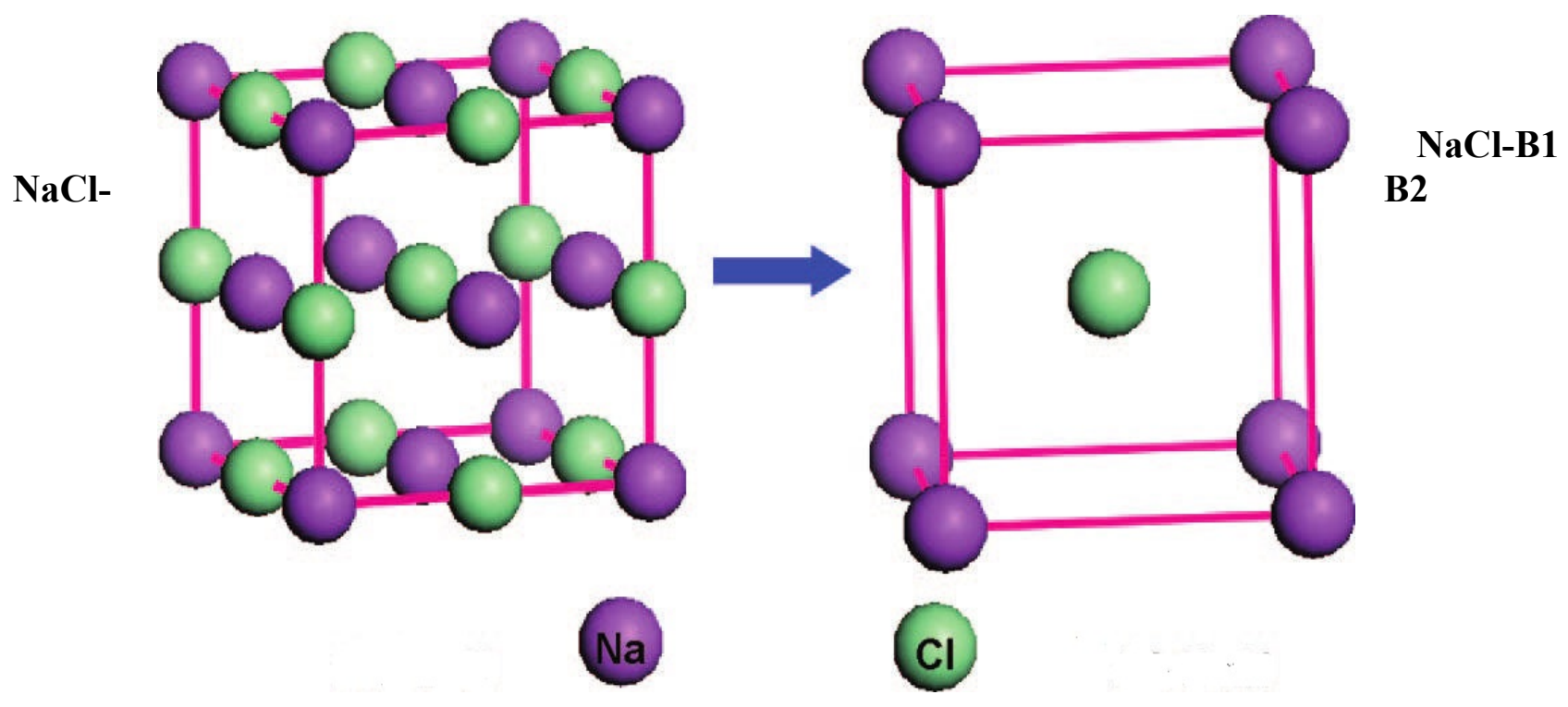

Fig. 1: Structures of $\mathrm{B} 1$ and $\mathrm{B} 2$ phases of $\mathrm{NaCl}$ (Lu et al., 2008). 
Table 1: Values of Bulk modulus at atmospheric pressure $K_{\mathrm{o}}$ and its First pressure derivative $K_{\mathrm{o}}^{\prime}$ for NaCl-B1.

\begin{tabular}{|l|l|l|}
\hline$K_{\mathrm{o}}$ (Gpa) & $K_{\mathrm{o}}^{\prime}$ (Gpa) & References \\
\hline 24 & 5.5 & Chauhan and Singh, 2007 \\
\hline 23.5 & 5.35 & Taravillo et al., 1996 \\
\hline 23.88 & 5.20 & Birch, 1986 \\
\hline 23.97 & 5.01 & Boehler and Kennedy, 1980 \\
\hline 23.8 & 4.0 & Sorensen, 1983 \\
\hline 24.0 & 5.38 & Demarest and Jr., 1972 \\
\hline
\end{tabular}

\section{THEORETICAL DETAILS}

This section presents two equations of state used with all thermoelastic parameters that are investigated under high pressure in the present work for $\mathrm{NaCl}-\mathrm{Bl}$.

\section{Equations of state (EOS):}

\section{- Dodson EOS:}

(Dodson, 1987) suggested simple two parameter EOS given as

$$
P_{D o}=\frac{27}{8} K_{\mathrm{o}} K_{\mathrm{o}}^{\prime^{2}}\left[\left(\frac{V}{V_{\mathrm{o}}}\right)^{-2 / 3}-1+4\left\{1-\frac{2}{3 K_{\mathrm{o}}^{\prime}}\right\} \times\left\{1-\left(\frac{V}{V_{\mathrm{o}}}\right)^{-1 / 3}-\frac{1}{6}\left(1-\frac{2}{3 K_{\mathrm{o}}^{\prime}}\right) \operatorname{Ln}\left(\frac{V}{V_{\mathrm{o}}}\right)\right\}\right]
$$

Where

$P_{D o}$-Pressure evaluated by using Dodson EOS, V-volume under high pressure $P, V_{\mathrm{o}}$-volume under atmospheric pressure. The relation, although empirical, fits all metals and metal alloys tested by (Dodson, 1987), and also fits semiconductors, ionic crystals, and with a lesser degree of success, organic compounds.

- Thomsen EOS:

Depending on the finite strain concept, where strains are uniquely determined by hydrostatic pressure. (Thomsen, 1970), suggested an EOS for solids in the form

$$
P_{\text {Tho }}=\frac{3 K_{\mathrm{o}}}{2}\left[\left(\frac{V}{V_{\mathrm{o}}}\right)^{-1 / 3}-\left(\frac{V}{V_{\mathrm{o}}}\right)^{1 / 3}\right]\left[1+\frac{3}{4} K_{\mathrm{o}}^{\prime}\left(1-\left(\frac{V}{V_{\mathrm{o}}}\right)^{2 / 3}\right)\right]
$$

Where

$P_{T h o}$ - high pressure evaluated by using Thomsen EOS.

\section{Bulk Modulus}

An important aspect of the EOS is the bulk modulus defined as

$$
K=-V\left(\frac{d P}{d V}\right)_{T}
$$

On the derivation of equations (1 and 2) with respect to volume and substituting in to eq.(3). Equations (4 and 5) give a variation of bulk modulus with high pressure by using Dodson EOS $\left(K_{D o}\right)$ and Thomsen $\operatorname{EOS}\left(K_{T \text { ho }}\right)$ as 


$$
\begin{aligned}
& K_{D o}=\frac{27}{8} K_{\mathrm{o}} K_{\mathrm{o}}^{\prime 2}\left[\frac{2}{3}\left(\frac{V}{V_{\mathrm{o}}}\right)^{-2 / 3}-4\left(1-\frac{2}{3 K_{\mathrm{o}}^{\prime}}\right)\left\{\frac{1}{3}\left(\frac{V}{V_{\mathrm{o}}}\right)^{-1 / 3}-\frac{1}{6}\left(1-\frac{2}{3 K_{\mathrm{o}}^{\prime}}\right)\right\}\right] \\
& K_{T \text { ho }}=\frac{K_{\mathrm{o}}}{2}\left[\left(\frac{V}{V_{\mathrm{o}}}\right)^{-1 / 3}+\frac{3}{4} K^{\prime}\left(\frac{V}{V_{\mathrm{o}}}\right)^{-1 / 3}+\frac{3}{2} K_{\mathrm{o}}^{\prime}\left(\frac{V}{V_{\mathrm{o}}}\right)^{1 / 3}+\left(\frac{V}{V_{\mathrm{o}}}\right)^{1 / 3}-\frac{9}{4} K_{\mathrm{o}}^{\prime}\left(\frac{V}{V_{\mathrm{o}}}\right)\right]
\end{aligned}
$$

\section{- Lattice Parameter}

Pressure dependence of lattice parameter of a solid is determined by (Radi et al., 2007) depending on Murnaghan EOS (Murnaghan, 1937) as:

$$
a=a_{\mathrm{o}}\left(1+K_{\mathrm{o}}^{\prime} \frac{P}{K_{\mathrm{o}}}\right)^{\frac{-1}{3 K_{o}^{\prime}}}
$$

Where

$a_{\mathrm{o}}$ and $a$ are lattice parameters at atmospheric pressure and under high pressure P respectively.

But the term $\left(1+K_{\mathrm{o}}^{\prime} \frac{P}{K_{\mathrm{o}}}\right)^{\frac{-1}{K_{o}^{\prime}}}$ in Murnaghan EOS is equivalent to $\frac{V}{V_{\mathrm{o}}}$.

Hence equation (6) can be rewritten as

$$
a=a_{\mathrm{o}}\left(\frac{V}{V_{\mathrm{o}}}\right)^{1 / 3}
$$

\section{- Grüneisen parameter}

(Boehler and Ramakrishnan, 1980) expressed pressure dependence of Grüneisen parameter by the following relation.

$$
\gamma_{P}=\gamma_{\mathrm{o}}\left(\frac{V}{V_{\mathrm{o}}}\right)^{q}
$$

\section{Where}

$\gamma_{\mathrm{o}}, \gamma_{P}$ - Grüneisen parameter at atmospheric pressure and under high pressure respectively. $q$ - second Gruneisen parameter. $q$ has been considered of an equal unity or a constant value.

But (Jeanloz, 1989; Agnon and Bukowiniski, 1990), suggested that $q$ could be changed under high pressure as:

$$
q=q_{\mathrm{o}}\left(\frac{V}{V_{\mathrm{o}}}\right)^{n}
$$

Where

$q_{\mathrm{o}}$ and $q$ are

second Grüneisen parameters at atmospheric pressure and under high pressure respectively, with $(n)$ as an equal unity or a positive number.

- Debye temperature $\left(\theta_{D}\right)$

Debye temperature $\left(\theta_{D}\right)$ or Debye characteristic temperature is a characteristic of each substance appearing in Debye theory of specific heats and given by: 


$$
\theta_{D}=\frac{\hbar \omega_{D}}{k_{B}}
$$

Where

$\theta_{D}$ - is the Debye temperature at atmospheric pressure, $k_{B}$ - Boltzmann constant, $\omega_{D}$ - Debye frequency at an atmospheric pressure.

Where a solid is considered as an elastic continuum whose maximum vibrational frequency is such that the corresponding number of vibrational modes is equal to the total number of degrees of freedom. As vibrational frequencies depend on equilibrium position which is changed with the pressure as a result of $\mathrm{V} / \mathrm{V}_{\mathrm{o}}$ variation with the pressure. (Preston et al., 1962) expressed Debye temperature under high pressure as

$$
\theta_{P}=\theta_{D}\left(\frac{V}{V_{\mathrm{o}}}\right)^{-\gamma_{P}}
$$

Where

$\theta_{P}$ - Debye temperature under high pressure. While (Decker, 1971) expressed Debye temperature as a function of Grüneisen parameter as:

$$
\theta_{P}=\theta_{D} \exp \left(\left(\gamma_{\mathrm{o}}-\gamma_{P}\right) / q\right)
$$

\section{CALCULATION AND RESULTS}

\section{- Calculation of $\frac{V}{V_{\mathrm{o}}}$ for NaCl-B1 using Dodson and Thomsen EOSs.}

On substituting values of $\mathrm{K}_{\mathrm{o}}$ and $K_{\mathrm{o}}^{\prime}$, the first row of Table (1), in Dodson EOS, eq. (1) one time, and in Thomsen EOS, eq. (2) another time. (Fig. 2) shows the results of variation of $V / V_{o}$ with high pressure obtained by using Dodson and Thomsen EOSs in comparison with the theoretical and experimental data published in the scientific literature.

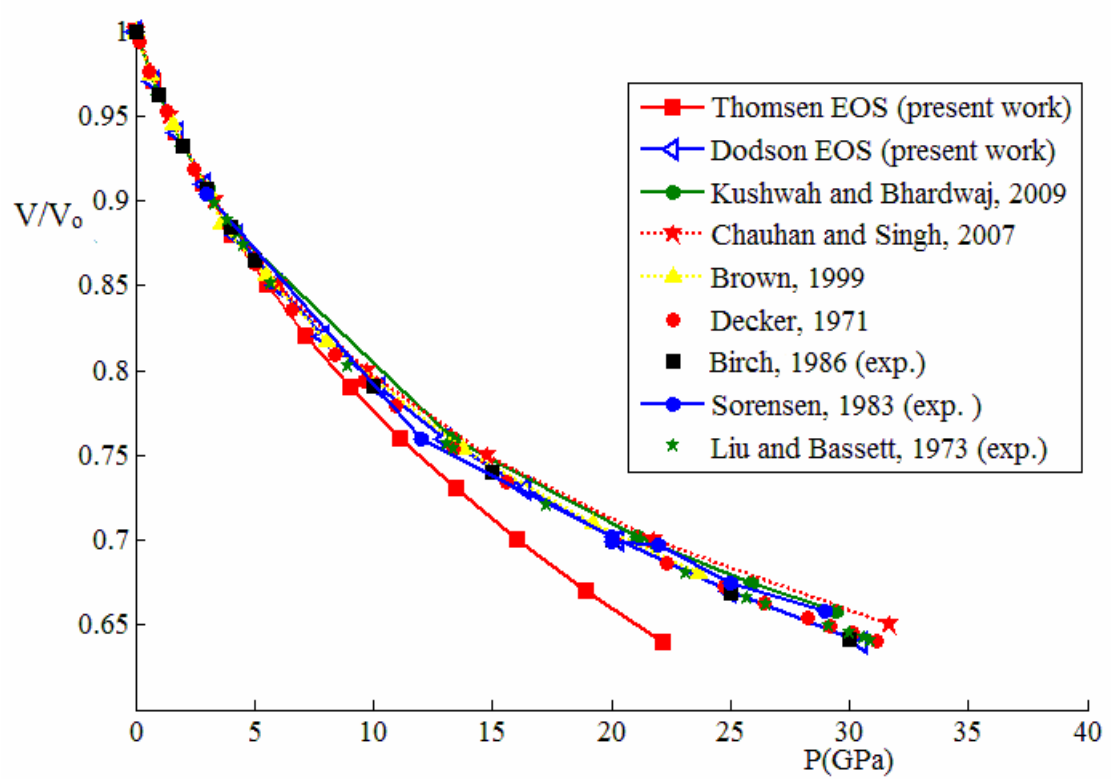

Fig. 2: Variation of $\mathrm{V} / \mathrm{V}_{\mathrm{o}}$ with high pressure for NaCl-B1, by using Dodson and Thomsen EOSs, in comparison with other works. 
Evaluation of bulk modulus $K$ under high pressure for NaCl-B1, using Dodson and Thomsen EOSs.

Substituting values of $K_{\mathrm{o}}$ and $K_{\mathrm{o}}^{\prime}$ from Table (1-first row) and $\mathrm{V} / \mathrm{V}_{\mathrm{o}}$ from (Fig. 2 ) in to eq. (4) and eq. (5), give values of bulk modulus, $\left(K_{D o}\right)$ and $\left(K_{T h o}\right)$ at different pressures corresponding to different $\mathrm{V} / \mathrm{V}_{\mathrm{o}}$ of (Fig. 2). The Obtained results are shown in (Fig. 3), in comparison with the experimental and theoretical data of different literature.

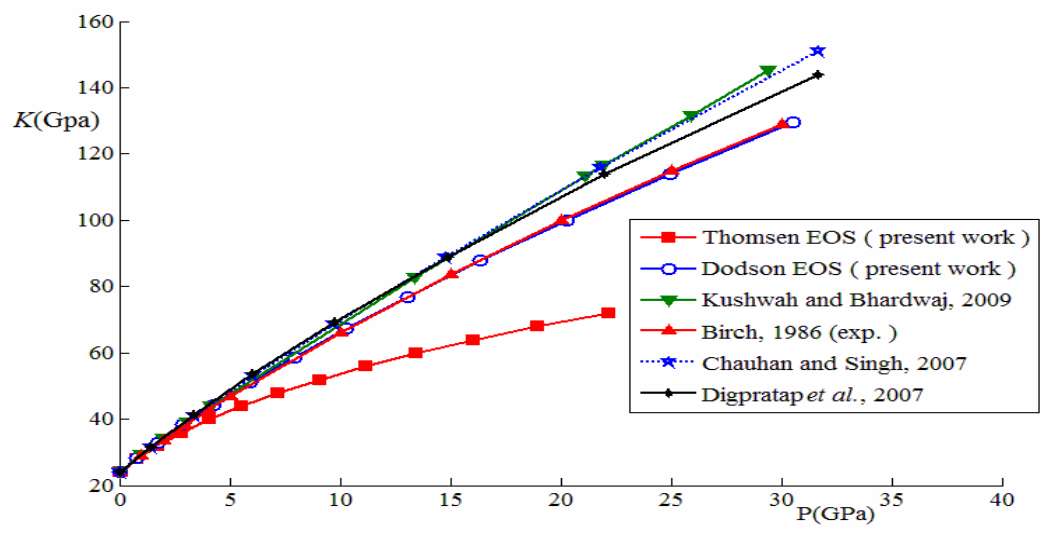

Fig. 3: Variation of bulk modulus $K$, for NaCl-B1, with high pressure, using Dodson and Thomsen EOSs, in comparison with experimental and theoretical data published in literature.

\section{Evaluation of Lattice parameter $a$ for NaCl-B1 under high pressure}

On combining ( $a_{0}=5.6402$ ) (Bassett et al., 1968), with $\mathrm{V} / \mathrm{V}_{\mathrm{o}}$ results from (Fig. 2), in equation (7). Lattice parameter $a$ has been evaluated at different pressures by using Dodson EOS one time and Thomsen EOS another time. (Fig. 4) shows the variation of NaCl-B1 lattice parameter with high pressure in comparison with the experimental data.

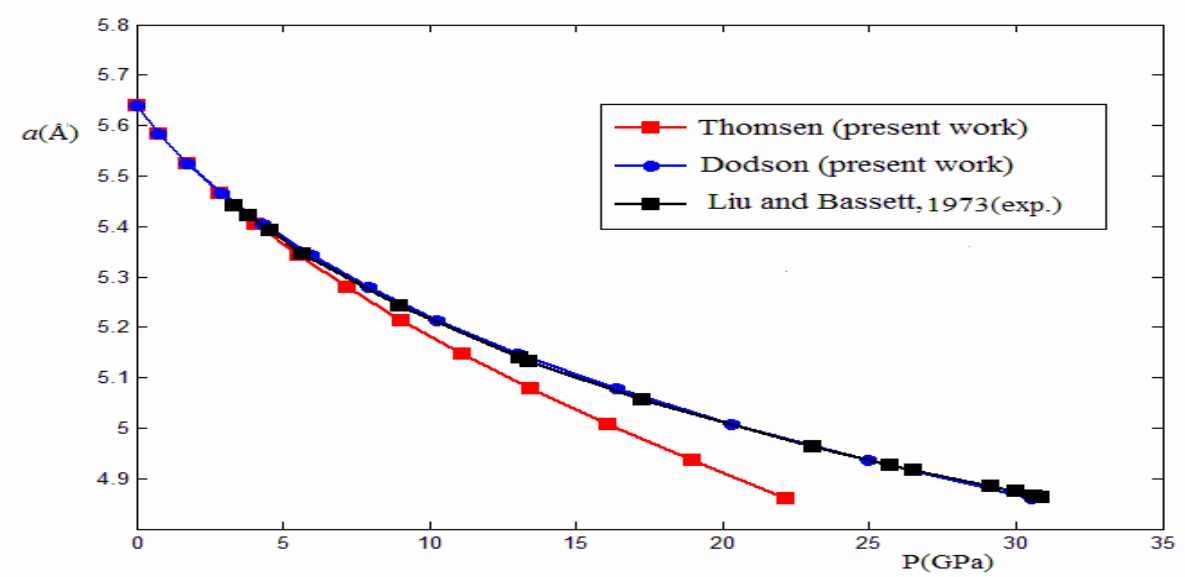

Fig. 4: Variation of NaCl-B1 lattice parameter $a$ with high pressure using Dodson and Thomsen EOSs in comparison with experimental data.

Evaluation of first Grüneisen parameter $\gamma_{P}$ for NaCl-B1 under high pressure using Dodson and Thomsen EOSs.

On substituting the experimental value of the Grüneisen parameter $\gamma_{0}$ from Table (2)(Birch,1986), and the value of second Grüneisen parameter $q_{o}$ from (Roberts and Ruppin, 1971), 
using $\mathrm{V} / \mathrm{V}_{\mathrm{o}}$ results shown in (Fig. 2) - (Dodson and Thomsen values) in to equation (8) (considering $q$ as a volume independent parameter and $q=q_{o}=1.4$ ), gives the results, for variation of first Grüneisen parameter $\gamma_{P}$ with the pressure as shown in (Fig. 5A).

On the other hand, combining equations (8 and 9) with $\mathrm{V} / \mathrm{V}_{\mathrm{o}}$ values of (Fig. 2)- (Thomsen and Dodson values) and recalculate the variation of $\gamma_{P}$ parameter of $\mathrm{NaCl}-\mathrm{B} 1$ with the pressure. (Fig. 5B) shows the values of $\gamma_{P}$ variation with high pressure by considering the volume dependence of second Grüneisen parameter $q$.

Table 2: Values of the first Grüneisen parameter $\gamma_{\mathrm{o}}$ and second Grüneisen parameter $q_{\mathrm{o}}$ for NaCl-B1

\begin{tabular}{|l|l|l|}
\hline$\gamma_{\mathrm{o}}$ & $q_{\mathrm{o}}$ & References \\
\hline 1.62 & & Birch, 1986 \\
\hline 1.61 & 1.4 & Roberts and Ruppin, 1971 \\
\hline 1.62 & 1.3 & Boehler and Ramakrishnan, 1980 \\
\hline
\end{tabular}

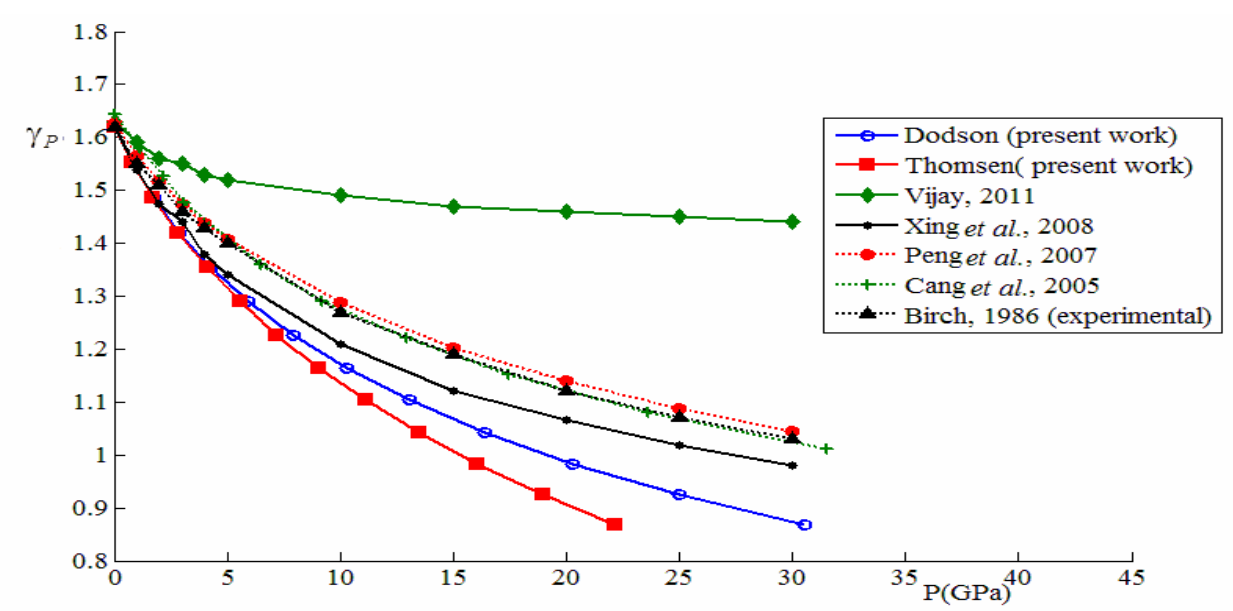

(A)

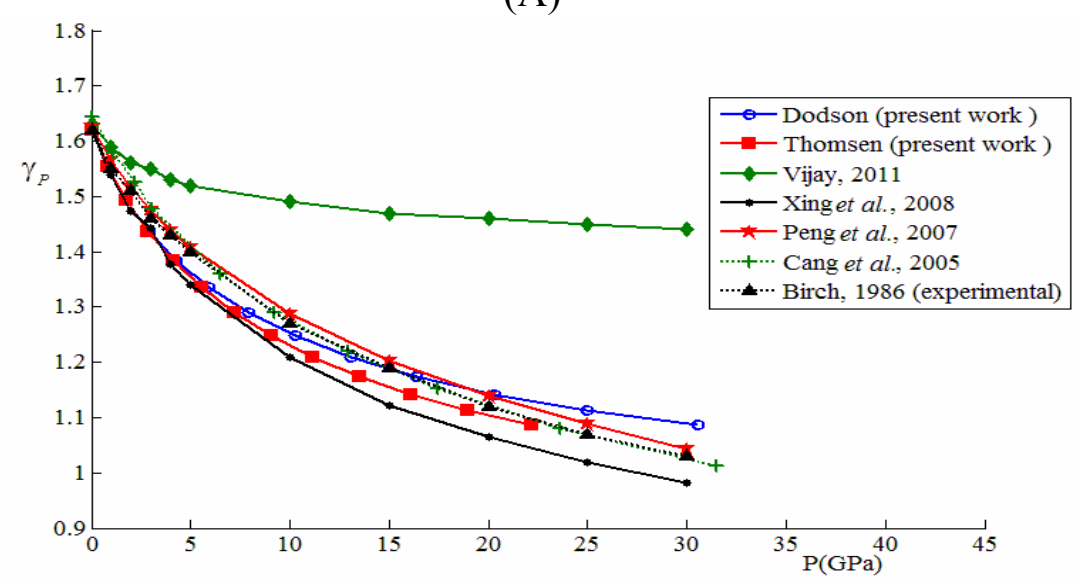

(B)

Fig. 5: Variation of first Grüneisen parameter of NaCl-B1 under high pressure by using Dodson and Thomsen EOSs, in comparison with data of other works.

A: $q$ is volume independent parameter.

B: $q$ is volume dependent parameter.

- Evaluation of Debye Temperature $\theta_{P}$ of NaCl-B1 under high pressure using Dodson and Thomsen EOSs. 
- Case 1: Grüneisen parameter $\left(\gamma_{\mathrm{o}}\right)$ is volume independent:

Using $\left(\theta_{D}=320\right)$ (Swenson, 1985), and value of Grüneisen parameter $\left(\gamma_{P}=\gamma_{\mathrm{o}}=1.62\right)$ - (Birch, 1986) and Substituting $\mathrm{V} / \mathrm{V}_{\mathrm{o}}$ values from (Fig. 2) (Thomsen and Dodson ), in to eq.(11) which could be rewritten as

$$
\theta_{P}=\theta_{D}\left(\frac{V}{V_{\mathrm{o}}}\right)^{-\gamma_{\mathrm{o}}}
$$

Fig. (6A) shows the variation of $\frac{\theta_{P}}{\theta_{D}}$ with high pressure using Dodson EOS one time and Thomsen EOS another time, in comparison with the theoretical data of (Fang, 2000).

- Case 2: Grüneisen parameter $\gamma_{P}$ is a volume dependent:

On considering the variation of Grüneisen parameter $\gamma_{P}$ with high pressure according to eq.(8) and eq.(9), with $q$ as a volume dependent parameter, combining them with equation (11), and substituting $\mathrm{V} / \mathrm{V}_{\mathrm{o}}$ from Fig. (2) (Thomsen and Dodson values) at different pressures. (Fig. 6B) shows the variation of $\frac{\theta_{P}}{\theta_{D}}$ with high pressure on considering the pressure dependence of $\gamma_{P}$ and $q$ parameters.

Similarly, the calculations for $\left(\theta_{P}\right)$ by using equation (12) are combined with $\left(\gamma_{P}\right.$ and $q-$ eq.8, 9$)$ give results shown in (Fig. 6B) with a less agreement with (Fang, 2000) data.

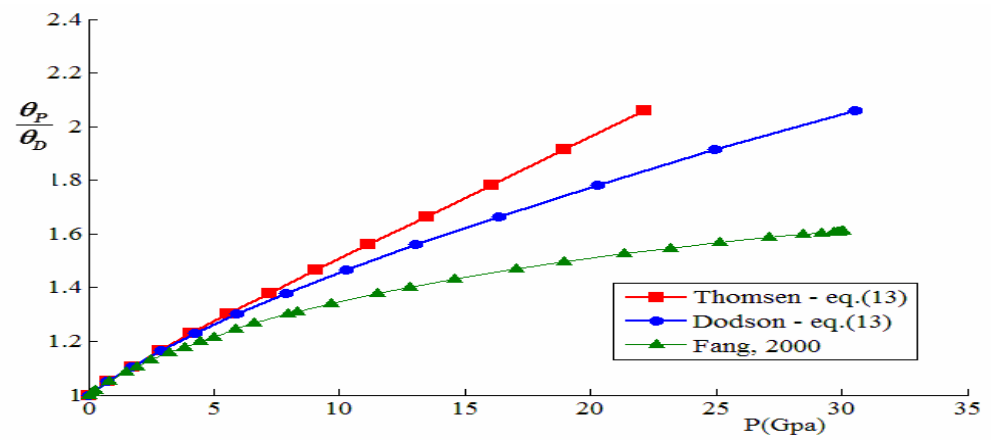

(A)

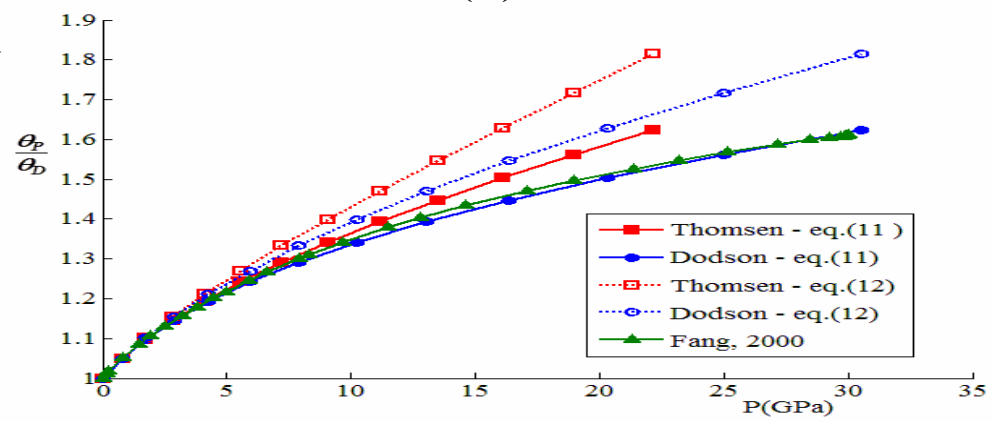

(B)

Fig. 6: Variation of $\frac{\theta_{P}}{\theta_{D}}$ of NaCl-B1, under high pressure using Dodson and Thomsen

EOSs, with in comparison with theoretical data of (Fang, 2000).

A: Grüneisen parameter $\gamma$ is a volume independent.

B: Grüneisen parameter $\gamma$ is a volume dependent.

\section{DISCUSSION}

1- The results of all works, including the present work, on variation of $\left(\mathrm{V} / \mathrm{V}_{\mathrm{o}}, K, a\right.$, and $\left.\theta_{P}\right)$ are in complete agreement under high pressure up to $3 \mathrm{GPa}$. While results for $\gamma_{P}$ are in less agreement. 
2- The results obtained, in the present work, using Dodson EOS show more agreement than that obtained by using Thomsen EOS with all data of other works. This may be attributed by the facts that Thomsen EOS based on finite strain, while Dodson EOS based on density functional theory which give it more generality.

3- On evaluation of variation of $\gamma_{P}$, for the case where second Grüneisen parameter $q$ is volume independent, both Dodson and Thomsen EOSs give results of less agreement with the data of other works Fig.(5A). In considering volume dependent of second Grüneisen parameter $q$ as represented in eq. (9), both Dodson and Thomsen EOSs give results which show a good agreement with the data of other works especially with the experimental data of (Birch, 1986) as shown in Fig. (5B).

Fig. (5-A, B) show that the results given by (Vijay, 2011) cannot match with the results obtained in the present work, for $\gamma_{P}$, or with any data of other works.

4- On considering variation of Debye temperature with high pressure, for the case where Grüneisen parameter $\gamma$ is volume independent, both Dodson and Thomsen EOSs give results of less agreement with that of other works (Fig. 6A). These results, especially that obtained by using Dodson EOS, improved and show a very good agreement with the data of (Fang, 2000) in considering Grüneisen parameter $\gamma_{P}$ to be volume dependent as represented in equation (11).

\section{REFERENCES}

Agnon, A.; Bukowinski, M.S.T. (1990). Thermodynamic and elastic properties of a manybody model for simple oxides. Phys. Rev.B, 41(11), 7755-7766.

Bassett, W.A.; Takahashi, T.; Mao, H.; Weaver, J.S. (1968). Pressure Induced Phase Transformation in NaCl. J. Appl. Phys., 39(1), 319-325.

Birch, F. (1986). Equation of State and Thermodynamic Parameters of $\mathrm{NaCl}$ to $300 \mathrm{kbar}$ in the High -Temperature Domain. J. Geophy. Res., 91(B5), 4949 - 4954.

Boehler, R.; Ramakrishnan, J. ( 1980 ). Experimental results on the pressure dependence of the Grüneisen parameter: A review. J. Geophy. Res., 85 (B12), 6996-7002.

Boehler, R.; Kennedy, G.C. (1980). Equation of State of Sodium Chloride up to 32k bar and 500C . J. Phys. Chem. Solids, 41, 517-523.

Brown, J. M. (1999). The NaCl pressure standard. J. Appl. Phys., 86(10), 5801-5808.

Cang, C.L.; Feng, C.Q.; Xin, C.S.; Qian, J.F. (2005). The Grüneisen parameter of $\mathrm{NaCl}$ at high pressures and temperatures: a Molecular dynamics study. Chin. Phys. Lett., 22(2), 514516.

Chauhan, R.S.; Singh, C.P. (2007). Equation of state and thermal expansivity of $\mathrm{NaCl}$ under high pressure and high temperature. Physica B, 387, 352-357.

Chen, Q.; Cai, L.; Duan, S.; Chen, D. (2004). Molecular dynamics simulation of thermodynamic properties of $\mathrm{NaCl}$ at high pressures and temperatures. J. Phys. Chem. Solids, 65, 1077-1081.

Decker, D.L. (1971). High Pressure Equation of State for $\mathrm{NaCl}, \mathrm{KCl}$ and $\mathrm{CsCl}$.J. Appl. Phys., 42(8), 3239-3244.

Demarest, H.H.; Jr. (1972). Extrapolation of elastic properties to High pressure in the Alkali Halides. J. Geophys. Res. 77(5),848-856.

Digpratap, S.; Sushil, K.; Singh, B.P. (2007). Pressure-volume-temperature relationship for diatomic solids based on inverted and non -inverted equations of state. Indian J. P. Appl. Phys., 45, 721-725.

Dodson, B.W. (1987-II). Universal scaling relations in compressibility of solids. J. Phys. Rev. B., 35(6), 2619-2625.

Fang, Z.H. (2000). On the pressure dependence of the Debye temperature for solids. Phys. Stat. Sol. (b), 222,451-456.

Jeanloz, R. (1989). Shock wave equation of state and finite strain theory. J. Geophy. Res., 94(B5), 5873-5886. 
Klotz, S.; Besson, J.M.; Böhning, S.M.; Nelmes, R.J.; Braden, M. (1995). Phonon dispersion measurements at high pressures to $7 \mathrm{GPa}$ by inelastic neutron scattering. Appl. Phys. Lett., 66,1557-1559.

Kumar, M.; Kumar, M. (2007). Shanker formulation needs modification at high pressures. J. Phys. Chem. Solids, 68, 670-672.

Kushwah, S.S.; Bhardwaj, N.K. (2009). Analysis based on equation of state for sodium halides. J. Phys. Chem. Solids, 70, 700-7002.

Liu, L.; Bassett, W.A. (1973). Compression of Ag and phase transformation of NaCl. J. Appl. Phys., 44, 1475-1479.

Lu, C.; Kuang, X.Y.; Zhu, Q.S. (2008). Characterization of the high-pressure structural transition and thermodynamic properties in Sodium Chloride: A computational investigation on the basis of the density functional theory. J. Phys. Chem. B, 112, 13898-13905.

Murnaghan, F.D. (1937). Finite deformation of an elastic solid. AM. J. Math., 49, 235-260.

Peng, X.C.; Xing, L.L.; Fang, Z.H. (2007).Comparing research on the Pressure or volume dependence of Grüneisen parameter. Physica B, 394,111-114.

Preston, R.S.; Hanna S.S.; Helerle J. (1962). Mössbaur effect in metallic Iron. Phys. Rev., 128(5), 2207-2218.

Radi, I.O.; Abdulsatter, M.A.; Abdul-Lettif, M. (2007). Semiemperical LUC- INDO calculations on the effect of pressure on the electronic structure of diamond. Phys. Stat. Sol. b, 244(4), 1304-1317.

Recio, J.M.; Pendas, A.M.; Francisco, E.; Florez, M.; Luana, V. (1993). Low- and high- pressure ab initio equations of state for the alkali chlorides. Phys. Rev. B, 48(9), 5891-5901.

Roberts, R.W.; Ruppin, R. ( 1971).Volume Dependence of the Grüneisen Parameter of Alkali Halides. Phys. Rev. B, 4(6), 2041- 2046.

Sorensen, Y.S. (1983). Phase Transitions and Equations of State for the Sodium Halides: NaF, NaC1, NaBr, and NaI. J. Geophy. Res., 88(B4), 3543- 3548.

Sun, X.W.; Wang, X.G.; Song, T.; Li, Y.H.; Liu, Y.X.; Chen, Q.F. (2008). Comparative investigations of the $\mathrm{P}-\mathrm{V}-\mathrm{T}$ relationship of $\mathrm{NaCl}$ at high pressure and temperature. Physica $B, \mathbf{4 0 3}, 3255-3260$.

Swenson, C.A. (1985). Volume dependence of the Grüneisen parameter: Alkali metals and $\mathrm{NaC} 1$. Phys. Rev. B, 31(2), 1150-1152.

Taravillo, M.; Baonza, V.G.; Nunez, J.; Caceres, M. (1996). Simple equation of state for solids under compression. Phys. Rev. B. 54 (10), 7034-7045.

Thomsen, L. (1970). On the fourth-order anharmonic equation of state of solids. J. Phys. Chem. Solids, 31, 2003-2016.

Ueda, Y.; Matsui, M.; Yokoyama, A.; Tange, Y.; Funakoshi, K. I. (2008). Temperature-pressurevolume equation of state of the B2 phase of sodium Chloride). J. Appl. Phys.103, 113513,1-6.

Vijay, A. (2011). Analysis of Grüneisen Parameter and its volume derivatives for $\mathrm{NaCl}$ and hcp iron. Indian J. P. Appl. Phys., 49, 825-828.

Xing, L.L.; Peng, X.C.; Fang, Z.H. (2008). Pressure and volume dependence of Grüneisen parameter of solids. J. Phys. Chem. Solids, 69, 2341- 2343. 